\title{
Multiple colon carcinomas in a patient with Cowden syndrome
}

\author{
ANJA-KATRIN BOSSERHOFF ${ }^{1}$, ELKE-INGRID GRUSSENDORF-CONEN ${ }^{3}$, \\ ALBERT RÜBBEN $^{3}$, SABINE RUDNIK-SCHÖNEBORN $^{2}$, KLAUS ZERRES ${ }^{2}$, \\ REINHARD BUETTNER ${ }^{4}$ and SABINE MERKELBACH-BRUSE ${ }^{4}$ \\ ${ }^{1}$ Institute of Pathology, University of Regensburg, 93053 Regensburg; ${ }^{2}$ Institute of Human Genetics and \\ ${ }^{3}$ Department of Dermatology, University Hospital RWTH, 52074 Aachen; ${ }^{4}$ Institute of Pathology, \\ University Hospital, 53127 Bonn, Germany
}

Received March 22, 2006; Accepted May 29, 2006

\begin{abstract}
Cowden syndrome is a non-adenomatous gastrointestinal polyposis syndrome with inactivation of PTEN, a dual-phosphatase tumor suppressor gene. Patients with loss of wildtype PTEN expression from one allele carry an increased risk of malignant breast, thyroid and brain tumors. However, the risk of malignant transformation in gastrointestinal polyps is still unclear. In this study, we describe a kindred with Cowden syndrome and identify a heterozygous germline mutation causing truncation of the PTEN tumor suppressor. The index patient was a 56 year-old woman having multiple facial papules, acral keratosis, oral papillomatosis, multiple benign breast and thyroid tumors and gastrointestinal polyposis. Progression to invasive adenocarcinoma occured in two pre-existing hamartomatous polyps. Analysis of one of the carcinomas revealed somatic inactivation of the wildtype PTEN allele by exon-skipping. This case demonstrates that gastrointestinal hamartomas in Cowden syndrome patients can progress to invasive adenocarcinomas and should therefore be carefully monitored.
\end{abstract}

\section{Introduction}

The inherited hamartoma syndromes were classified by Eng and Ji (1) into four clinical entities: Cowden syndrome (CS), Bannayan-Ruvalcaba-Riley syndrome (BRR), Peutz-Jeghers syndrome (PJS), and juvenile polyposis syndrome (JPS). In patients with CS and BRR $(2,3)$, inherited germline mutations in the PTEN tumor suppressor gene were identified. PTEN, located on chromosome 10q23, encodes a dual-specificity phosphatase that modulates cell cycle progression and cell survival by regulating phosphatidylinositol 3,4,5,-triphosphate and the Akt/protein kinase B signaling pathway (4).

Correspondence to: Dr Anja K. Bosserhoff, Institute of Pathology, University of Regensburg, Franz-Josef-Strauss-Allee 11, 93053 Regensburg, Germany

E-mail: anja.bosserhoff@klinik.uni-regensburg.de

Key words: colon cancer, Cowden syndrome, germline mutation, PTEN
PTEN heterozygous mice exhibit neoplasms in multiple organs including the endometrium, liver, prostate, gastrointestinal tract, thyroid and thymus. Tumors of the gastrointestinal epithelium develop in association with lymphoid tissue. This finding is consistent with the model that PTEN behaves as a 'landscaper' in the colon (5). In humans, CS is characterized by multiple hamartomas of the skin, breast, thyroid, oral mucosa and gastrointestinal tract. Gastrointestinal polyps are present in up to $60 \%$ of patients with CS (4). Patients are reported to be at an increased risk for breast (30-50\% of patients) and thyroid cancer ( $10 \%$ of patients), however the risk of malignant transformation of the gastrointestinal polyps is still unclear (6).

In this study, we identified an inherited PTEN germline mutation in a family with an unusual manifestation of CS. Multiple colon carcinomas arose from hamartomatous polyps, and analysis of mRNA and protein revealed somatic inactivation of the wildtype PTEN allele in one of the tumors.

\section{Materials and methods}

Blood samples were obtained from the patient and two relatives. Extraction of total cellular RNA from the tumor tissue, extraction of genomic DNA from the tumor and blood samples, PCR, RT-PCR and cycle-sequencing were performed using standard procedures (7). The $1.2 \mathrm{~kb}$ PTEN cDNA was PCR amplified and sequenced using the following primers: forward, 5'-atgacagccatcatcaaagag-3'; reverse, 5'-tcagacttttg taatttgtg-3'. Bidirectional DNA sequencing of the entire PTEN coding region and exon-intron boundaries was performed on all blood samples using primers listed in Table I. The identity of the amplicon sequences was confirmed by database search (PTEN accession no. AF143314, NCBI database). To prove the biallelic origin of the detected mutations, cDNA from the tumor tissue was amplified in two separate reactions using the following PCR primers: Reverse mut, 5'-tatcattacaccagttca-3'; reverse wt, 5'-tatcattacacca gttcg-3'; forward, 5'-atgacagccatcatcaaaag-3'. PCR conditions were: $5 \mathrm{~min}$ at $94^{\circ} \mathrm{C}$, then 30 cycles of $1 \mathrm{~min}$ at $94^{\circ} \mathrm{C}, 45 \mathrm{sec}$ at $58^{\circ} \mathrm{C}, 1 \mathrm{~min}$ at $72^{\circ} \mathrm{C}$ and finally $5 \mathrm{~min}$ at $72^{\circ} \mathrm{C}$.

Protein was extracted from tumor tissue of the Cowden patient and two other colon carcinomas using RIPA lysis 
Table I. Primers used for genomic sequencing.

\begin{tabular}{lll}
\hline Exon & \multicolumn{1}{c}{ Sense primer (5'-3') } & Antisense primer (5'-3') \\
\hline 1 & cagcagcttctgccatctc & atatgacctagcaacctgacca \\
2 & tgaccaccttttattactcca & aagtatctttttctgtgtggctt \\
3 & atagaaggggtatttgttgga & acctcactctaacaagcagata \\
4 & ttcaggcaatgtttgtta & ctcgataatctggatgactca \\
5 & gcaacatttctaaagttaccta & tctgttttccaataaattctca \\
6 & agtgaaataactataatggaaca & gtaaacttctagatatggtta \\
7 & atcgttttgacagtttg & atagcttttaatctgtcc \\
$8 \mathrm{~A}$ & aggtgacagattttctttttta & cagctgtactcctagaatta \\
$8 \mathrm{~B}$ & aggtgacagattttctttttta & cttcatcagctgtactcc \\
$9 \mathrm{~A}$ & gttcatctgcaaaatgga & tggtaatctgacacaatgtccta \\
9B & attaatatgttcatctgc & tggtaatctgacacaatgtccta \\
\hline
\end{tabular}

buffer (Roche, Mannheim, Germany), subjected to SDSPAGE on $4-20 \%$ gradient polyacryamide gels (Invitrogen, Groningen, Netherlands) and transferred onto PVDF membranes. The membranes were probed with an anti-PTEN antibody (Santa Cruz, CA, USA, 1:100) and visualized by alkaline phosphatase conjugated goat-anti-mouse IgG followed by color development with NBT and BCIP. Sections of the tumor tissue, hamartomatous polyps and the corresponding control sections were examined immunohistochemically with anti-PTEN (Santa Cruz, CA, dilution 1:40) and anti-p-Akt (Cell Signaling, Frankfurt, Germany, dilution 1:50) antibodies.

\section{Results}

Case presentation. The 56 year-old index patient came to the dermatology department for the removal of her cutaneous tumors. She had multiple trichilemmomas, acral keratosis and oral papillomas. At the age of 20 she underwent a partial thyroidectomy, from which histological data are not available. The diagnosis of CS was established at the age of 56 when the patient developed two independent adenocarcinomas of the ileocecal valve and the ascending colon. The patient's gastrointestinal tract showed hamartomatous polyposis extending from the esophageous to the colon. Resection of the right hemicolon and terminal ileum was perfomed revealing numerous small hyperplastic polyps in the small and large intestines as well as two different adenocarcinomas at the ileocecal valve and in the ascending colon (Fig. 1A). Histologically, the polyps were composed of hyperplastic crypts with lymphofollicular infiltration of the stroma. Microglandular mucinous adenocarcinomas arose within these hyperplastic polypous lesions, and extended metastasis had occured in the regional lymph nodes (Fig. 1B-D).

The patient's younger sister had similar mucocutaneous manifestations with predominating lipomas on arms and legs.
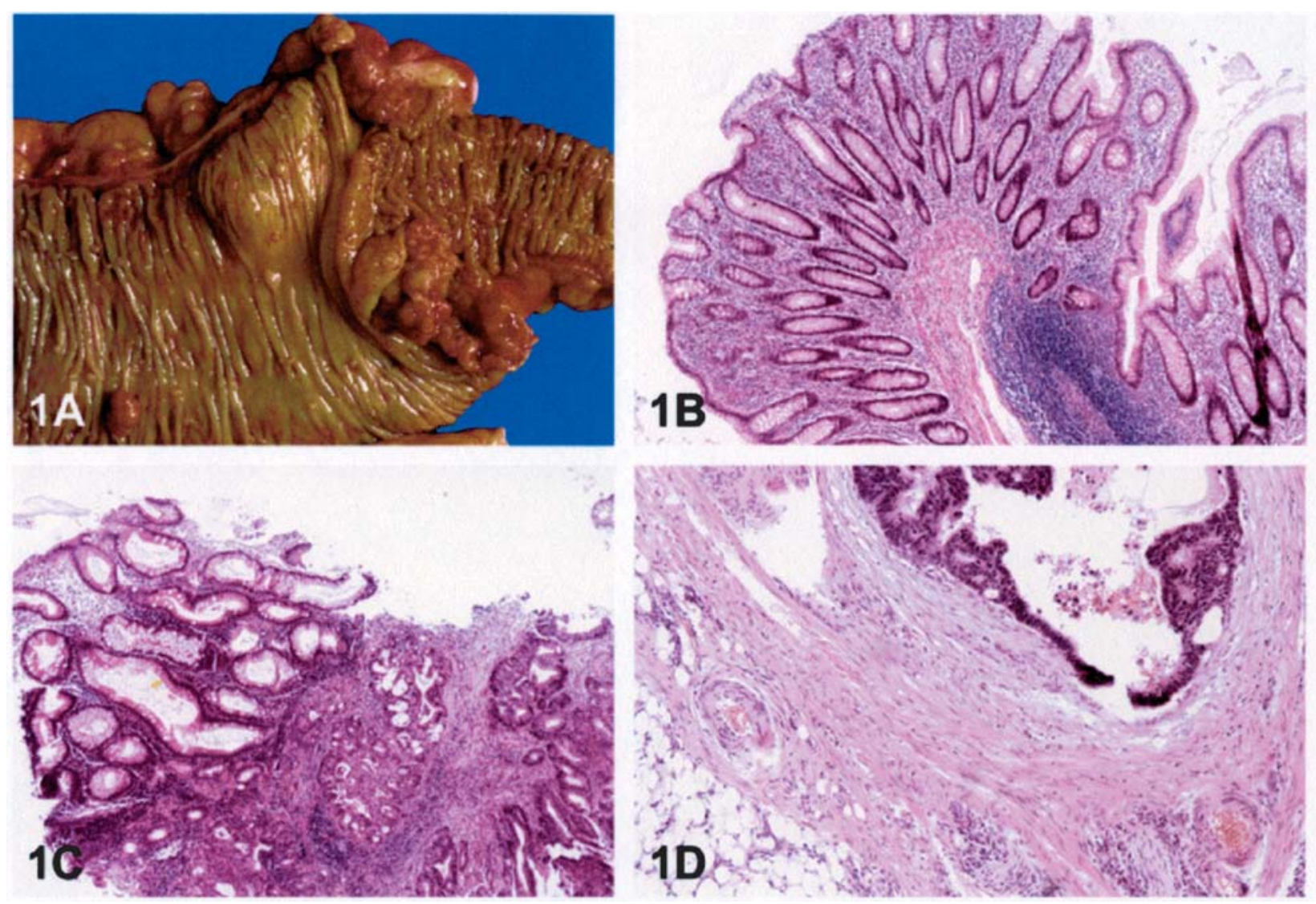

Figure 1. Hamartomatous polyposis with colon carcinomas in Cowden syndrome. (A) Macroscopic view of the hemicolectomy showing a large tumor at the ileocecal valve and a second tumor in the ascending colon as well as numerous small polyps in the ileum and colon. (B) Microscopic view of a hyperplastic polyp with lymphofollicular infiltration in the colon (H\&E, x100). (C) Histological section of the tumor at the ileocecal valve showing glandular adenocarcinoma arising in a hyperplastic polyp (H\&E, x100). (D) Metastasis in a regional lymph node (H\&E, x250). 


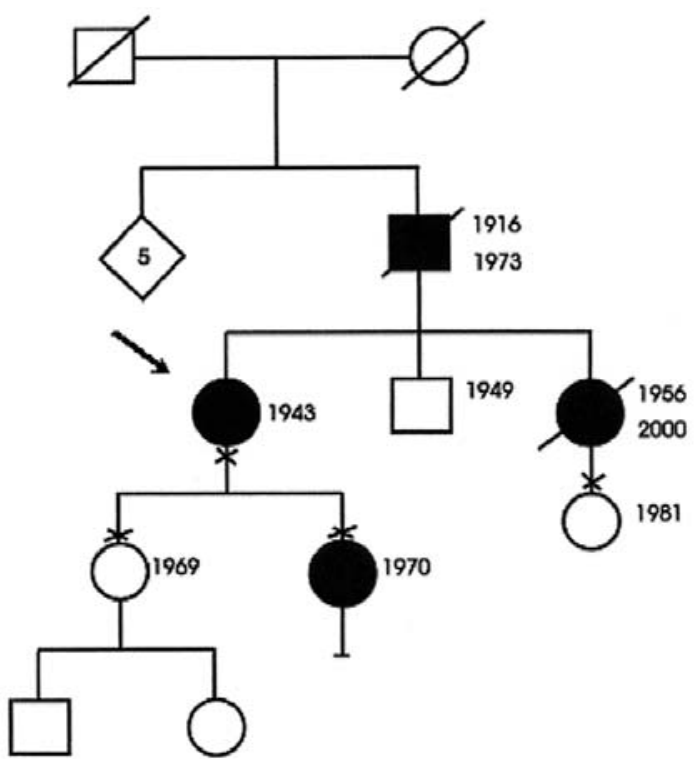

A
A G G GAC GA AC T

proband (1943)

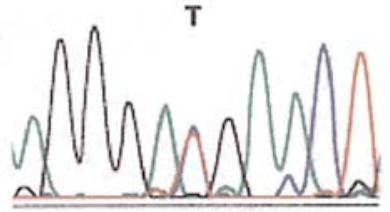

A G G G A C A A C T

affected

daughter (1970)

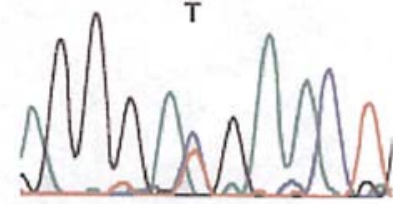

A G G GAC GAACT

healthy

daughter

(1969)

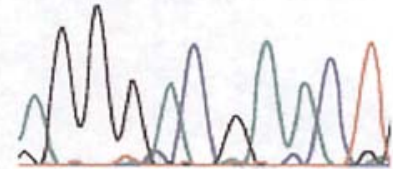

B

Figure 2. Pedigree of the Cowden family (A). Sequence analysis of peripheral blood DNA from the Cowden patient and the two daughters. C-T transition results in a stop mutation (B).

A

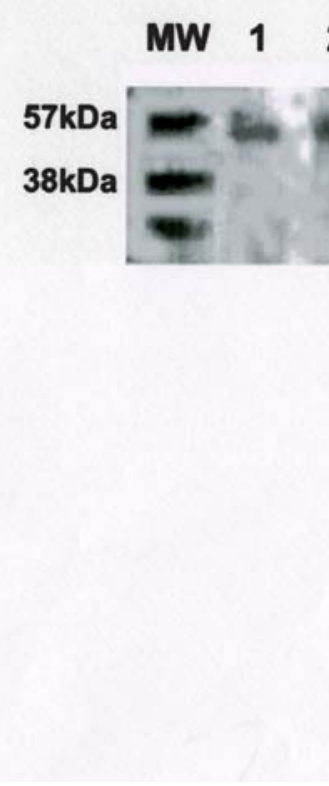

B

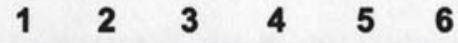

$47 \mathrm{kDa}$

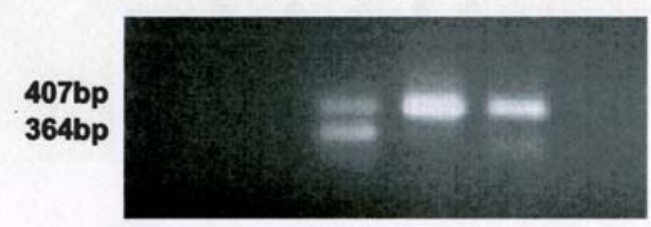

C

C T T T G T G C T G A

heterozygot

loss of Exon 4

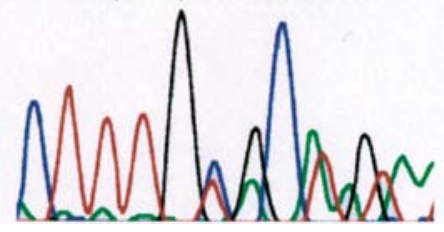

Figure 3. Somatic inactivation of the PTEN gene in the tumor tissue. (A) Western-blot analysis using anti-PTEN-antibody comparing protein extracts from the Cowden patient $(1,2)$ with extracts from sporadic colon carcinomas $(3,4)$. (B) Sequence analysis of PTEN mRNA showing exon-skipping of exon 4 . PCR analysis confirmed the biallelic origin of both mutations. Lane 1 and 2, negative controls; lane 3 and 4, cDNA from the Cowden patient; lane 5 and 6 , cDNA of a sporadic colon carcinoma. (C) Sequence analysis of RNA of the tumor tissue confirmed skipping of exon 4.

She suffered from Hashimoto thyreoiditis but had no malignant disease. Her gastrointestinal tract had not been examined until she died suddenly at the age of 44 . The cause of death remained unknown. The father had multiple cutaneous tumors and died at the age of 57 after several strokes. The second daughter of the index patient had facial papillomas, acral keratosis and nodular goiter with histologically confirmed follicular adenomas. She underwent a thyroidectomy at the age of 31. All affected family members (Fig. 2A) who were available for examination had megalencephaly while none of the relatives without mutations had an increased head circumference. 
LOH and PTEN mutation analysis. $\mathrm{LOH}$ analysis was performed with DNA extracted from the colon carcinoma and corresponding germline DNA from peripheral blood using a microsatellite marker flanking PTEN (D10S541, Research Genetics, Huntsville, AL, USA). The marker showed heterozygosity for both the tumor and normal DNA. Direct sequencing of the patient's peripheral blood DNA revealed a heterozygous $\mathrm{C}-\mathrm{T}$ transition at the first nucleotide of codon 130 resulting in the substitution of a stop codon for ARG (R130X). Upon further analysis of the family, we found that one of the patient's daughters had inherited the mutated allele (Fig. 2B).

Immunohistochemistry and Western blot. Western blot analysis using an anti-PTEN antibody was performed on protein samples of the Cowden patient's colorectal carcinoma and two sporadic colon carcinomas. The approximately $47 \mathrm{kDa}$ PTEN band was detected only in the two sporadic carcinomas, leading to the conjecture of somatic PTEN inactivation in the tumor tissue (Fig. 3A). The approximately $55 \mathrm{kDa}$ band detected in all samples was due to unspecific binding of the antibody. These findings were confirmed by immunohistochemistry. PTEN immunostaining of the tumor tissue and the gastrointestinal polyp was completely negative in contrast to internal control sections and positive pAkt reactions (data not shown).

Biallelic inactivation of PTEN. Direct sequencing of PTEN using genomic DNA from the patient's tumor did not reveal any additional mutation. To further analyze the cause of somatic inactivation of the PTEN gene in the tumor tissue, the $1.2 \mathrm{~kb}$ PTEN mRNA was amplified by RT-PCR. Sequence analysis showed skipping of the entire exon 4 resulting in a frameshift of all downstream exons (Fig. 3B). To prove the biallelic origin of the PTEN mutations, mRNA was amplified in two separate PCR reactions using two different reverse primers. The mutant reverse primer hybridizes only to mutated exon 5 sequences whereas the wt reverse primer anneals to wildtype sequences. As shown in Fig. 3C, PCR amplification with the mutant reverse primer yielded only a 407 bp fragment containing exon 4, whereas amplification with the wildtype primer produced a $364 \mathrm{bp}$ fragment devoid of exon 4 . The $407 \mathrm{bp}$ fragment in the latter reaction was due to the contamination of the tumor tissue with normal tissue.

\section{Discussion}

Cowden syndrome is classified as a familial hamartomatous polyposis syndrome and its association with histopathologically different types of gastrointestinal polyps has been observed (4). The analysis of PTEN germline mutations in patients with CS provides evidence that PTEN functions as a tumor suppressor in this disease and related heritable disorders (2). In the present study we analyzed a family with an unusual manifestation of CS. A germline PTEN mutation (R130X) leading to a stop codon in exon 5 was identified in two members of the CS family including our index patient. This nonsense mutation directly affects the phosphatase core motif which encompasses codons 123 to 131 and is regarded as a mutational hot spot (8).
According to Knudson, tumor suppressor genes such as $\mathrm{Rb}$ have been shown to undergo 'two-hit' inactivation in sporadic as well as in familial cancers. We analyzed the colorectal carcinoma and the gastrointestinal polyps from our CS index patient for this second hit. By $\mathrm{LOH}$ analysis we were able to show that the remaining PTEN wildtype allele was not deleted. Sequence analysis of mRNA extracted from tumor tissue revealed the exon-skipping of exon 4 . A causal splice site mutation at the genomic level could not be detected. A possible explanation is that only the exons and exon-intron boundaries were sequenced. In $85 \%$ of cases, splice site mutations are responsible for exon-skipping, but unknown mutations possibly located in the intron account for the remaining $15 \%$ (9). Exon-skipping of exon 4 results in instable mRNA and protein with strongly reduced dual phospahatase activity (10-12).

The biallelic origin of the described mutations was proven by PCR analysis using two different PCR primer sets. Further evidence for the complete inactivation of both PTEN alleles came from immunohistochemistry and Western-blot analysis.

Biallelic inactivation of PTEN due to different mechanisms is common in several sporadic malignant tumors, including melanoma, glioblastoma and endometrial carcinoma $(13,14)$. In other tumor types, e.g. primary breast and prostate carcinomas, hemizygous deletions have been found which may be sufficient for tumorigenesis as has been demonstrated with hemizygous PTEN knock-out mouse models $(5,14)$. In patients with CS, PTEN germline mutations accompanied by somatic loss of the wildtype allele have been described for benign lesions including breast fibroadenoma, thyroid adenoma, pulmonary hamartoma and different types of gastrointestinal polyps $(15,16)$. To our knowledge biallelic PTEN inactivation in CS-associated carcinomas has not yet been described perhaps due to the focusing on $\mathrm{LOH}$ analysis by several groups (15). As described in this study, the second hit may also be represented by small mutations or mechanisms causing transcript destabilization.

In conclusion, the detection of germline mutation, the retention of the mutant allele in tumor samples of the CS index patient and the somatic mutation of the wildtype allele provide further evidence that PTEN encodes a tumor suppressor. Malignant transformation in Cowden syndrome patients likely occurs in a variety of different organs possibly influenced by modifier genes or environmental factors.

\section{References}

1. Eng C and Ji H: Molecular classification of the inherited hamartoma polyposis syndromes: clearing the muddied waters. Am J Hum Genet 62: 1020-1022, 1998.

2. Liaw D, Marsh DJ, Li J, Dahia PL, Wang SI, Zheng Z, Bose S, Call KM, Tsou HC, Peacocke M, Eng C and Parsons R: Germline mutations of the PTEN gene in Cowden disease, an inherited breast and thyroid cancer syndrome. Nat Genet 16: 64-67, 1997.

3. Marsh DJ, Dahia PL, Zheng Z, Liaw D, Parsons R, Gorlin RJ and Eng C: Germline mutations in PTEN are present in BannayanZonana syndrome. Nat Genet 16: 333-334, 1997.

4. Starink TM, van der Veen JP, Arwert F, de Waal LP, de Lange GG, Gille JJ and Eriksson AW: The Cowden syndrome: a clinical and genetic study in 21 patients. Clin Genet 29: 222-233, 1986.

5. Podsypanina K, Ellenson LH, Nemes A, Gu J, Tamura M, Yamada KM, Cordon-Cardo C, Catoretti G, Fisher PE and Parsons R: Mutation of Pten/Mmac1 in mice causes neoplasia in multiple organ systems. Proc Natl Acad Sci USA 96: 1563-1568, 1999. 
6. Carlson GJ, Nivatvongs S and Snover DC: Colorectal polyps in Cowden's disease (multiple hamartoma syndrome). Am J Surg Pathol 8: 763-770, 1984.

7. Bosserhoff AK, Moser M, Hein R, Landthaler M and Buettner R: In situ expression patterns of melanoma-inhibiting activity (MIA) in melanomas and breast cancers. J Pathol 187: 446-454, 1999.

8. Bonneau D and Longy M: Mutations of the human PTEN gene. Hum Mutat 16: 109-122, 2000.

9. Nakai K and Sakamoto H: Construction of a novel database containing aberrant splicing mutations of mammalian genes. Gene 141: 171-177, 1994.

10. Celebi JT, Wanner M, Ping XL, Zhang $\mathrm{H}$ and Peacocke M: Association of splicing defects in PTEN leading to exon skipping or partial intron retention in Cowden syndrome. Hum Genet 107: 234-238, 2000.

11. Agrawal S and Eng C: Differential expression of novel naturally occurring splice variants of PTEN and their functional consequences in Cowden syndrome and sporadic breast cancer. Hum Mol Genet 15: 777-787, 2006.

12. Agrawal S, Pilarski R and Eng C: Different splicing defects lead to differential effects downstream of the lipid and protein phosphatase activities of PTEN. Hum Mol Genet 14: 2459-2468, 2005 .
13. Zhou XP, Gimm O, Hampel H, Niemann T, Walker MJ and Eng C: Epigenetic PTEN silencing in malignant melanomas without PTEN mutation. Am J Pathol 157: 1123-1128, 2000.

14. Kurose K, Zhou XP, Araki T and Eng C: Biallelic inactivating mutations and an occult germline mutation of PTEN in primary cervical carcinomas. Genes Chromosomes Cancer 29: 166-172, 2000.

15. Marsh DJ, Dahia PL, Coulon V, Zheng Z, Dorion-Bonnet F, Call KM, Little R, Lin AY, Eeles RA, Goldstein AM, Hodgson SV, Richardson AL, Robinson BG, Weber HC, Longy M and Eng C: Allelic imbalance, including deletion of PTEN/MMACI, at the Cowden disease locus on 10q22-23, in hamartomas from patients with Cowden syndrome and germline PTEN mutation. Genes Chromosomes Cancer 21: 61-69, 1998.

16. Chi SG, Kim HJ, Park BJ, Min HJ, Park JH, Kim YW, Dong SH, Kim BH, Lee JI, Chang YW, Chang R, Kim WK and Yang MH: Mutational abrogation of the PTEN/MMAC1 gene in gastrointestinal polyps in patients with Cowden disease. Gastroenterology 115: 1084-1089, 1998. 